\title{
Propuesta de cadena de VAlor en la fabricación de PANELES FOTOVOLTAICOS
}

\author{
VALUE CHAIN PROPOSAL IN THE MANUFACTURE OF \\ PHOTOVOLTAIC PANELS \\ Dairon Rojas Hernández ${ }^{1}$ \\ Estela Gertrudis Espinosa Martínez ${ }^{2}$ \\ Arístides Pelegrín Mesa ${ }^{3}$ \\ doi.org/10.52292/j.eca.2021.2654
}

Fecha de recepción 29/04/2021

Fecha de aceptación 15/09/2021

\section{Resumen}

El proceso de transformaciones económicas que están ocurriendo en Cuba considera a la empresa como eje central en el mercado, para el desarrollo de actividades y la toma de decisiones. La investigación aborda los fundamentos teóricos relacionados con los encadenamientos productivos, como base de la cadena de valor y la cadena de valor en la industria. Dada la importancia que tiene la sustitución del consumo de combustibles fósiles en la generación de energía y en otras ramas de la economía, se propone el procedimiento para diseñar una cadena de valor de la fabricación de paneles fotovoltaicos en el país. Se utiliza una metodología con enfoque integral, utilizando diferentes métodos capaces de validar los resultados obtenidos, entre los que se destacan:

$1 \quad$ Universidad de Pinar del Río. E-mail: dairon920328@gmail.com. ORCID: https:/ / orcid. org/0000-0003-1443-6318

2 Universidad de La Habana. E-mail: estelaespinosa975@gmail.com. ORCID: https://orcid. org/0000-0003-2813-1785

$3 \quad$ Universidad de Guadalajara. E-mail: pelegrin65@yahoo.es. ORCID: https:/ / orcid.org/00000003-1230-1276 
el procedimiento diseñado, su validación en la empresa objeto de investigación, así como una derivación de estrategias empresariales para esta entidad.

Palabras clave: encadenamientos, cadena de valor, paneles fotovoltaicos.

\begin{abstract}
The process of economic transformations that are taking place in Cuba considers the company as a central axis in the market, for the development of activities and decision-making. The research addresses the theoretical foundations related to production chains, as the basis of the value chain and the value chain in the industry. Given the importance of replacing the consumption of fossil fuels in power generation and in other branches of the economy, the procedure is proposed to design a value chain for the manufacture of photovoltaic panels in the country. Where a methodology with a comprehensive approach is used, using different methods, capable of validating the results obtained, among which the following stand out: the designed procedure, its validation in the company under investigation, as well as a derivation of business strategies for this entity.
\end{abstract}

Keywords: chains, value chain, photovoltaic panels.

JEL: M40, M41, M49. 


\section{Introducción}

En el contexto actual de la economía mundial la cadena de valor es pertinente como herramienta para diseñar e implementar las estrategias empresariales, frente a un entorno saturado de oferentes, con constantes cambios del mercado y el juicio cada vez más exigente de los consumidores. Esto es debido a que, como herramienta estratégica, la cadena se orienta hacia la creación de valor para los clientes, mediante el fortalecimiento e impulso de aquellas actividades o competencias distintivas que permiten generar una ventaja competitiva.

El tejido de relaciones que se establecen en un sistema de producción, comercialización y acceso al mercado constituye la base para las cadenas de valor. Sin embargo, la filosofía intrínseca en esta última parte del intercambio y colaboración en la información entre los actores supone, tanto dentro como fuera de la misma, que contribuyan al desarrollo de sistemas y productos para la satisfacción de los clientes de una forma tal que, una vez que se logra, es muy difícil imitar por la competencia. Todo ello, fue desarrollado ampliamente por Michel Porter en la década de los ochenta del siglo XX en su obra Competitive Advantage: Creating and Sustaining Superior Performance (Porter, 1985), bibliografía que fue tomada como base para esta investigación, así como trabajos posteriores del propio autor, donde resalta la importancia de la cadena de valor como herramienta para la planificación estratégica.

Cuba se encuentra en un proceso de realización de transformaciones en su modelo económico iniciado en 2011, orientado por la Comisión de Implementación de Lineamientos del VI Congreso del Partido Comunista de Cuba (PCC) celebrado en el año 2012, que define la cadena de valor como la “etapa superior en el desarrollo de la cadena productiva para crear o potenciar capacidades productivas competitivas y productos de mayor valor agregado y contenido científico-tecnológico [...]" (Partido Comunista de Cuba [PCC], 2012). Dichos lineamientos se han ido perfilando en la Conceptualización del Modelo Económico y Social cubano, así como en el Plan de Desarrollo Económico y Social hasta el 2030, donde se hace un énfasis particular en propiciar los encadenamientos productivos a partir del desarrollo de nuevas formas de organización de la producción, basadas en la tecnología, la innovación, la gestión del conocimiento, la introducción de la ciencia en la producción y su sostenibilidad en el tiempo.

En este sentido de sus proyecciones estratégicas, Cuba se plantea la urgencia de la transformación de su matriz energética, para disminuir el consumo de combustibles importados para la generación de energía eléctrica, así como el 
desarrollo de fuentes renovables y el uso eficiente de la energía, lineamientos 14 y 202 respectivamente (PCC, 2016).

La empresa de Componentes Electrónicos de Pinar del Río es la encargada de la fabricación de paneles fotovoltaicos, para sustentar la utilización de energías renovables en el país, por lo que fue elegida para la investigación. Cuenta con diversas producciones destacando las actividades fundamentales que son la fabricación de paneles fotovoltaicos (la principal), consumibles electrónicos para respaldo y protección de línea y electrónica automotriz, que se dirigen al sector industrial y de los servicios para satisfacer el mercado interno y estar presentes en el mercado externo, con productos que se enmarcan en las ramas de las energías renovables, la electrónica, la informática y servicios para aplicaciones industriales.

La referida empresa es la única de su tipo en Cuba, la cual está ampliando la capacidad productiva de productos fotovoltaicos. Para la implementación de su estrategia de desarrollo, solicitó una colaboración científica a la Universidad de Pinar del Río y la Universidad de La Habana. En este caso, la identificación de la cadena de valor sería muy útil en la contribución a la proyección estratégica de la empresa y el mejoramiento de su desempeño.

Sin embargo, no tienen definidos los inputs y los outputs de los procesos, desde la entrada física de las materias primas hasta el producto terminado y su entrega al cliente. Tampoco están identificadas las actividades generadoras de valor en dichos procesos, todo lo cual está recogido en Rojas et al. (2020a) y Rojas et al. (2020b).

Las actividades de valor que decide realizar cualquier unidad de negocio son las que definen la estrategia competitiva o estrategia del negocio, diferentes a las estrategias corporativas o las estrategias de un área funcional (Bonmatí, 2011).

El empleo de la cadena de valor permitiría elevar, entonces, el nivel de cooperación industrial, así como la comunicación tecnológica entre las empresas tanto nacionales como internacionales, para aumentar la capacidad de investigación y desarrollo y fiabilidad del producto, como establece la Norma Internacional de Contabilidad N. ${ }^{\circ} 1$, Norma de Información Financiera N. ${ }^{\circ}$, así como en la Norma Específica N. ${ }^{\circ} 12$, por parte del Ministerio de Finanzas y Precios de Cuba.

La presente investigación va encaminada a mostrar una solución al siguiente problema: ¿cómo diseñar la cadena de valor en la fabricación de paneles fotovoltaicos, en la empresa de Componentes Electrónicos, en función de lograr un proceso de toma de decisiones efectivo que representen nuevas posibilidades para potenciar la competitividad? 
En consecuencia, el objetivo general del presente trabajo consiste en proponer el diseño de la cadena de valor en la fabricación de paneles fotovoltaicos en la empresa de Componentes Electrónicos.

Derivado del objetivo general, se desagregan los siguientes objetivos específicos:

1. Fundamentar los aspectos teórico-conceptuales relacionados con la cadena de valor en la industria.

2. Diagnosticar la situación existente de la empresa de Componentes Electrónicos respecto a la cadena de valor en la fabricación de paneles fotovoltaicos.

3. Diseñar la cadena de valor en la fabricación de paneles fotovoltaicos sobre la base de los fundamentos teórico-conceptuales asumidos y el diagnóstico realizado.

El trabajo está estructurado en seis apartados. En la introducción, se refleja la problemática a resolver y la relevancia de la investigación. Parte de un marco teórico conceptual sobre las cadenas productivas como base para la comprensión de las cadenas de valor y, en particular, la cadena de valor industrial. Se presenta un acápite para el tratamiento metodológico, el que es tomado como base para la propuesta de procedimiento para el diseño de la cadena de valor en la fabricación de paneles fotovoltaicos, que es el principal resultado del trabajo, que se desarrolla en el cuarto apartado. Finalmente, en la discusión de los resultados se señalan los aspectos del diseño que ya se han desarrollado preliminarmente, así como los que aún deben ser desarrollados. En las conclusiones, se resalta la importancia del tema para Cuba, en particular en la necesidad de potenciar el sector industrial y la sustitución de importación de combustibles, donde la cadena de valor para esta industria es clave.

\section{Marco teórico}

\subsection{La cadena de valor como herramienta analítica}

Cuando se hace alusión a la herramienta de la cadena de valor, las perspectivas de análisis pueden ser diversas, pero todas tienen como punto de partida a Michael Porter y su teoría de la ventaja competitiva sostenible; se trata de un método analítico muy útil y adaptable a cualquier empresa, actividad 
productiva o sector económico, y también a la dimensión territorial del desarrollo (Menoya, 2015, citado en Domínguez et al., 2017).

El tratamiento de la misma se centrará fundamentalmente en la cadena de valor industrial, que permite realizar un análisis interno de una industria mediante su desagregación en las principales actividades generadoras de valor, que se encuentran relacionadas o eslabonadas y representan el proceso a través del cual un producto recibe incorporaciones de valor.

Señala Porter (1993) que la ventaja competitiva de una empresa proviene del conjunto de actividades discretas que la empresa realiza en el diseño, producción, marketing, entrega y apoyo de su producto o servicio. Cada una de estas actividades puede contribuir a mejorar la posición competitiva basada en menores costos o a reforzar las bases para una mayor diferenciación. Por tanto, la cadena de valor es la herramienta que permite realizar un análisis sistemático y riguroso de esas actividades, desagregando la empresa en sus actividades estratégicamente relevantes con el fin de comprender mejor el comportamiento de los costos y de las fuentes existentes y potenciales de diferenciación.

Una cadena de valor comprende la amplia variedad de actividades requeridas para que un producto o servicio transite, a través de diferentes etapas, desde su concepción hasta su entrega a los consumidores y la disposición final después de su uso (Kaplinsky \& Morris, 2002, citado en Padilla \& Oddone, 2016). El concepto de cadena de valor comprende todas las etapas (eslabones) necesarias para que un bien o servicio transite de su concepción y diseño hasta su consumo y disposición final (Padilla \& Oddone, 2016). Los eslabones, por su parte, están constituidos por los actores que realizan alguna actividad relacionada con el proceso productivo del bien o servicio, su distribución, comercialización y, finalmente, su venta.

1 Las actividades básicas o primarias, que tienen que ver directamente con la creación de valor (logística de entrada, operaciones, logística de salida, marketing y servicio al cliente).

2. Las actividades de apoyo o secundarias, que sirven para que las primeras puedan coordinarse, administrarse, compartir información, etc. (infraestructura, recursos humanos, investigación-desarrollo y abastecimiento). 
Figura 1. Cadena de valor de Michael Porter

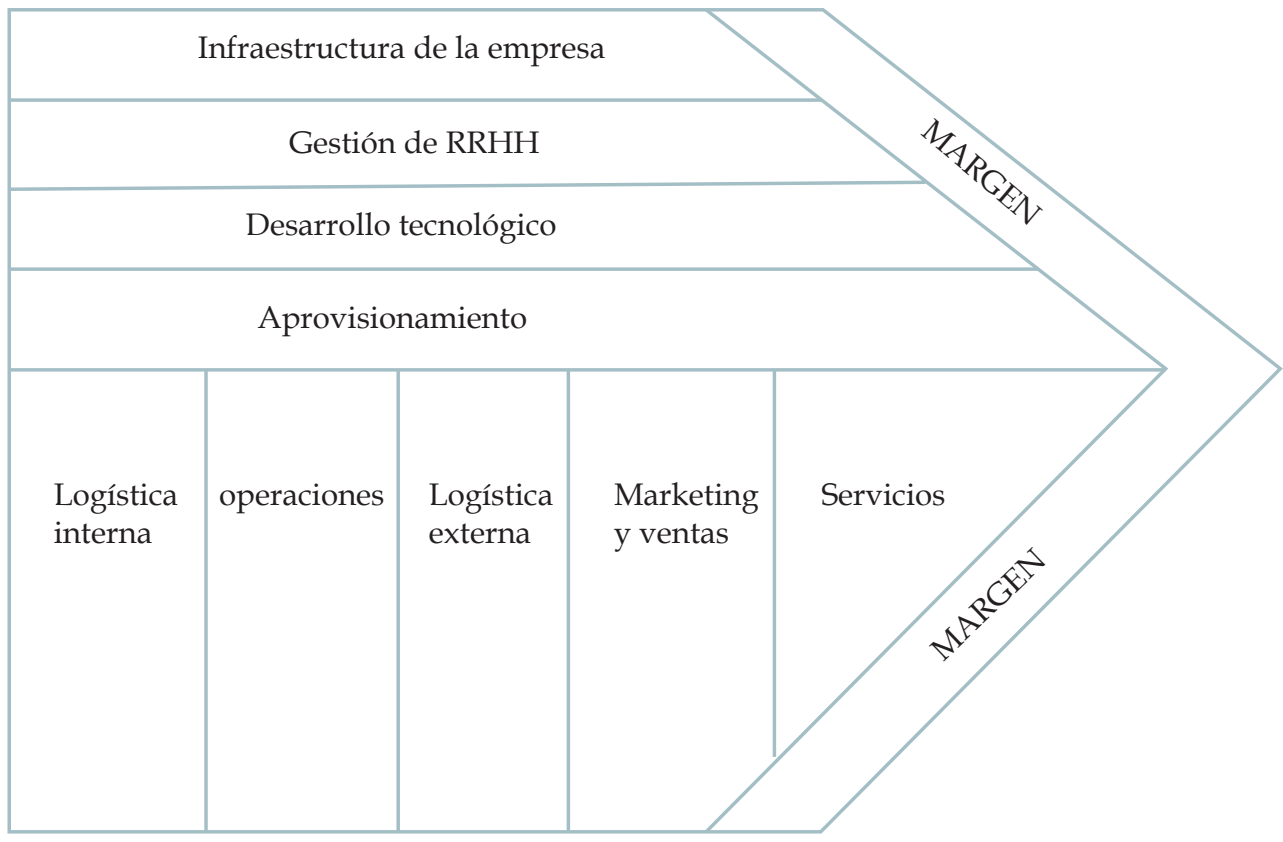

Fuente: Porter (1993).

\subsubsection{La cadena productiva como base de la cadena de valor}

El concepto de cadena productiva tiene sus inicios en los primeros trabajos que en 1958 realizó Albert Hirschman sobre el desarrollo económico (Isaza, 2005). Este economista propone que la existencia de encadenamientos de cooperación entre diferentes organizaciones explica los mayores niveles de generación de riqueza en las economías industrializadas del primer mundo. La clave de tales encadenamientos reside, fundamentalmente, en la capacidad de articular acuerdos o contratos de cooperación que facilitan y hacen más eficientes los procesos productivos (Blanco, 2013).

Siguiendo a López (2011), se entiende por cadena productiva la red social de actores que a través de una adecuada gestión de conocimiento se convierte en cadena de valor para generar resultados:

1. generar una visión estratégica compartida entre los actores de la cadena; 
2. viabilizar su orientación hacia la demanda;

3. propiciar una cultura de aprendizaje colaborativo que facilite la coinnovacción y la gestión óptima individual y colectiva de sus procesos productivos y;

4. mejorar los flujos de información y comunicación a lo largo de la cadena.

La integración de una cadena productiva en una cadena de valor enfatiza la vinculación entre las empresas participantes de cada eslabón, para establecer proyectos colectivos y de asociación empresarial que faciliten el acceso a nuevos mercados y permitan el progreso competitivo.

La cadena productiva no consiste en realizar las actividades independientemente, sino en buscar relacionarlas de tal manera que se hagan interdependientes. Cada eslabón de la cadena debe ser optimizado y coordinado con los demás para lograr ventajas competitivas (Porter, 1993, citado en Plasencia, 2006).

Para Van der Heyden y Camacho (2006), una cadena productiva es un sistema constituido por actores interrelacionados y por una sucesión de operaciones de producción, transformación y comercialización de un producto o grupo de productos en un entorno determinado.

En la figura 2 se expone un esquema conceptual, sencillo o simplificado, de cadena productiva según los autores Van der Heyden y Camacho (2006).

Figura 2. Esquema simplificado de cadena productiva

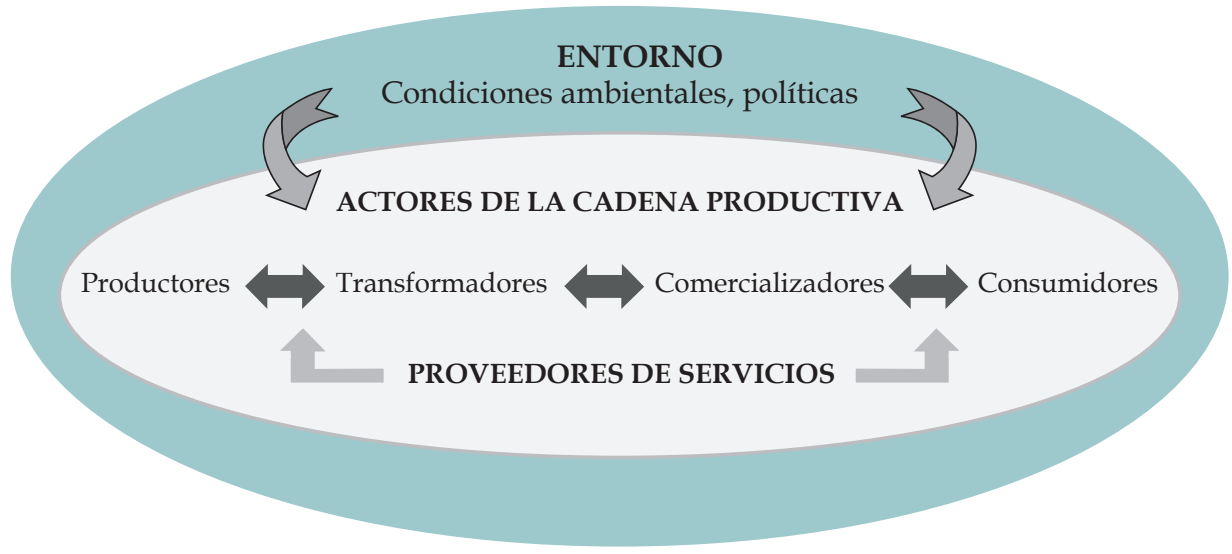

Fuente: Van der Heyden \& Camacho (2006). 
Las cadenas productivas reflejan una situación dinámica y una interacción espontánea, sus actores cambian y pueden pertenecer también a otras cadenas productivas independientes, su entorno evoluciona y se modifica, en ella intervienen muchos actores que mantienen relaciones complejas entre ellos, pueden existir cuellos de botellas, vacíos, traslapes de actividades y costos de transacción que restan competitividad y disminuyen sus beneficios. En la figura 3 se representa una cadena más cercana a la realidad, donde se puede apreciar la complejidad de las relaciones que pueden darse y las dificultades para su interpretación.

Figura 3. Esquema propuesto de cadena productiva

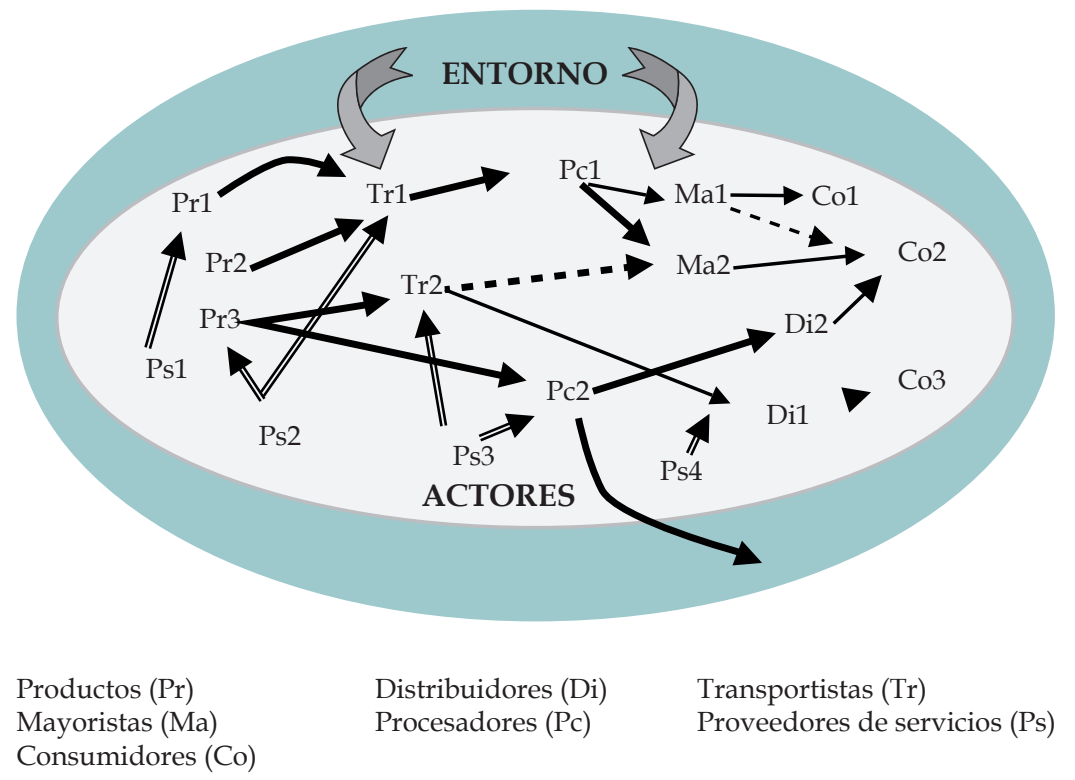

Fuente: Van der Heyden \& Camacho (2006).

Otros autores, como Wisner (2003) y Gorbaneff y Restrepo (2007), conceptualizan la cadena productiva como la integración de los procesos clave de negocios que ocurren dentro de la red, conformada por los proveedores de insumos, los fabricantes, los distribuidores y los minoristas independientes, cuyo objetivo es optimizar el flujo de los bienes, servicios e información.

Es decir que se trata de un concepto que involucra un número de etapas interconectadas, a través de la unión entre producción, transformación y 
consumo; lo que implica que se consideren los distintos procesos productivos y relaciones económicas que se producen entre la oferta inicial y la demanda final (Gottret, 2007).

La cadena productiva es un conjunto articulado de actividades económicas integradas como consecuencias de articulaciones en términos de mercado, tecnología y capital. En este sentido, se coincide con García (2007), que la entiende como el conjunto de agentes económicos que participan directamente en la producción, después en la transformación y en el traslado hasta el mercado de realización de un mismo producto; es el conjunto de actividades estrechamente interrelacionadas verticalmente, vinculadas por su pertenencia a un mismo producto y cuya finalidad es satisfacer al consumidor.

Según Vivanco et al. (2010), una cadena productiva se define como el proceso que sigue un producto o servicio a través de las actividades de producción, transformación e intercambio, hasta llegar al consumo final. Incluye, además, el abasto de insumos (financiamiento, seguros, maquinaria, equipo, materias primas directas e indirectas, etc.) y sistemas relevantes, así como todos los servicios que afectan de manera significativa a dichas actividades: investigación, desarrollo, capacitación y asistencia técnica, entre otros.

Desde principios del siglo XXl, se hace referencia a encadenamientos de procesos clave. Es por ello que, cuando se habla de cadena productiva, se está aludiendo al encadenamiento de procesos clave de negocio, que ocurren dentro de una cadena conformada por proveedores de insumos, fabricantes, distribuidores y minoristas independientes, con el objeto de optimizar el flujo de productos, bienes, servicios e información, que son ofrecidos y distribuidos a los consumidores para satisfacer la demanda (Espinal, 2004; Mandal, 2012).

En la literatura se ha descrito cómo, en la organización del proceso de producción y entrega del producto final al cliente, es importante que cada actor de la cadena o que la cadena productiva en sí misma genere valor para su comprador final. Este es uno de los elementos que le puede permitir generar una base para su diferenciación en el mercado y, de esta forma, asegurar la competitividad (Jiménez \& Montenegro, 2014).

La cadena productiva puede, en general, referirse al conjunto de actores, procesos y recursos interrelacionados e interdependientes que permiten que uno o varios productos lleguen al mercado final en un contexto determinado (Vinci, 2014).

López (2016) plantea la necesidad de distinguir entre cadena productiva y cadena de valor, ya que si la disponibilidad de un producto en el mercado depende de una cadena productiva, la sostenibilidad de esta cadena depende 
en gran medida de que el mercado adquiera de manera estable ese producto, $\mathrm{y}$, a su vez, depende del valor que el producto contiene. Por tanto, una cadena productiva debe organizarse y desarrollarse para generar valor y no simplemente productos.

Las experiencias que existen en Cuba de esta temática están centradas en industrias de tecnología avanzada (biotecnología e industria de medicamentos), el sector de la agricultura (el mango y la producción tabacalera), forestal (servicios ecosistémicos forestales), la industria alimentaria (cultivo del camarón y conservas de tomate) y el sector del turismo en Viñales para potenciar el desarrollo local (Menoya, 2015; Anaya, 2015; López, 2016; Rojas, 2017; Espinosa \& Díaz, 2021; Máttar \& Padilla, s. f.). Un elemento clave para las cadenas es quién ejerce el poder, pues este determina la manera en que se distribuye el valor generado (Anaya, 2015).

Nova et al. (2020) plantean que el concepto de encadenamiento debe ser entendido como una relación a largo plazo, que se establece entre unidades empresariales con el propósito de obtener beneficios conjuntos. Debe sustentarse en los beneficios económico-sociales que se derivan y no en el encadenamiento por sí mismo. Estos autores definen el encadenamiento productivo-valor, en términos generales, como el conjunto de actividades involucradas en el diseño, la producción y la comercialización de un producto o servicio. Los encadenamientos implican un grado de compromiso entre las empresas más allá de una relación de compra-venta normal. Una definición más abarcadora del encadenamiento puede ser aquella que se refiere a una amplia interrelación sectorial y/o geográfica de empresas que se desempeñan en las mismas actividades o en actividades estrechamente relacionadas. Tomando como referencia las relaciones establecidas en el sector agropecuario cubano en la década del noventa del pasado siglo, en el referido trabajo se tratan no solo los llamados vínculos "hacia atrás" (hacia los proveedores de insumos y equipos, entre otros) o "hacia delante" (actividades desarrolladas de forma conjunta o conectadas, acopio de productos, transportación, almacenamiento, comercialización, beneficio, procesamiento industrial), sino aquellos denominados vínculos "hacia los lados" (industrias procesadoras y usuarias, así como a servicios y actividades estrechamente relacionadas). En ese sentido, se consideran de esta categoría importantes actividades económicas fuera del marco municipal o provincial, que incluso pueden llegar a extenderse hasta las cadenas globales de valor externas en búsqueda de eficiencia colectiva (Nova et al., 2020). 
En resumen, para Nova et al. (2020), el enfoque de cadenas productivas encierra varias ventajas. Permite tener una visión más amplia de la cadena y de sus diferentes actores, por lo tanto, un manejo más completo de la información. Esto facilita la identificación de puntos críticos que impiden su desarrollo y la ubicación de alternativas más efectivas y de mayor impacto, logrando así mejor competitividad. Califica la cadena como un escenario apropiado para la búsqueda de alianzas y sinergias entre los diferentes actores productivos, ya que reúne actores con intereses comunes, lo cual disminuye los costos de interacción y permite un uso más eficaz de los recursos disponibles, ya sea a nivel territorial, más allá de límites municipales, provinciales o hasta la inserción externa.

Plantean Antúnez y Ferrer (2021) que el análisis de cadenas constituye una herramienta que permite identificar los puntos críticos que frenan la competitividad y las ventajas competitivas que potencian los encadenamientos, para luego definir e impulsar estrategias de acción concertadas entre los principales actores involucrados.

\subsubsection{Cadena de valor industrial}

Cuando Porter, en 1985, introdujo el concepto de la cadena de valor en sus estudios e investigaciones, fue para lograr identificar las actividades decisivas de la empresa; el objetivo de ese análisis era mejorar la rentabilidad que le generaba un mayor grado de competitividad a la misma (Manzo, s. f.). El estudio y análisis del concepto de cadena de valor continúa siendo de interés para los académicos y directivos de las organizaciones, ya que se percibe como una de las alternativas más exclusivas de todo el cúmulo de actividades que la empresa realiza y que reflejan el éxito y utilidades de los productos finales; además facilita la toma de decisiones permitiendo crear estrategias que generen ventajas competitivas.

Según la Organización de las Naciones Unidas para el Desarrollo Industrial (ONUDI, 2011) un marco integrado para el diagnóstico de la cadena de valor debe estar en condiciones de describir el estado de su desarrollo en una variedad de aspectos/dimensiones del diagnóstico. Revisando las prácticas comunes en el análisis de la cadena de valor es posible distinguir cuatro enfoques, en parte superpuestos: 
Dairon Rojas Hernández, Estela Gertrudis Espinosa Martínez, Arístides Pelegrín Mesa

a. Enfoques de gestión estratégica y administración de empresas que observan la gestión de la cadena de la oferta y el desarrollo de la empresa individual.

b. Enfoques de desarrollo del clúster industrial, en los cuales se asume que la organización espacial, alianzas empresariales estratégicas y la integración son el origen de la competitividad sistémica.

c. El enfoque de la cadena de valor global, que hace énfasis en los réditos económicos y estructuras de gobernabilidad, determinado, por ejemplo, por el predominio de compradores y minoristas que operan internacionalmente.

d. El enfoque de sistema de innovación, según el cual el acceso a conocimiento y tecnología, así como las oportunidades de utilizarlos, permite a los actores participar en la cadena de valor.

La fragmentación internacional de la producción industrial se está expandiendo a un ritmo acelerado (Timmer et al., 2014), dando lugar a procesos que tienen formas muy distintas e incluso complejas (Baldwin \& Venables, 2013). En la actualidad, el comercio y la producción mundiales se estructuran alrededor de las llamadas cadenas globales de valor (Organisation for Economic Co-operation and Development [OECD], 2012).

Lladós et al. (2018) plantean que el estudio de la cadena de valor nos permite identificar todo el conjunto de actividades que una industria necesita para llevar un producto final al mercado, desde su concepción hasta su uso por parte de los consumidores finales. Y, a su vez, refleja cuál es el valor añadido de todas las actividades que directa o indirectamente son necesarias para su producción.

También identifican las diferentes características de la industria de equipos eléctricos y electrónicos que inducen su protagonismo en el desarrollo de sistemas de producción y generación de valor. En realidad, las cadenas de producción globales son procesos sofisticados de generación de valor añadido, con cada integrante adquiriendo inputs y añadiendo valor al producto intermedio en forma de salarios y rentas de capital que, a su vez, constituirán los costos de la siguiente fase de producción.

La primera fase es la elevada modularización de sus productos. Los principales productos del sector y sus propios procesos productivos pueden ser ampliamente formalizados, codificados, estandarizados y computarizados, lo que facilita una gran interoperabilidad de las partes y componentes que, a su vez, propician la fragmentación del proceso productivo en diferentes fases 
o etapas. Es decir, el modularidad no solo permite modificar la arquitectura de los productos, también abre nuevas oportunidades para la organización de la industria (Sturgeon \& Kawakami, 2011). De este modo, tanto el diseño como la logística o cada una de las etapas de producción pueden ser implementadas por diferentes empresas localizadas en distintas ubicaciones (De Backer \& Miroudot, 2014).

La industria electrónica tiene un papel crucial en la configuración de dichas cadenas (Lladós et al., 2018). Tal y como afirma Nogueira (2012), es la cadena global de valor más dinámica y extensa geográficamente de todos los sectores productivos. La importancia de este proceso de organización internacional de la producción se refleja en el peso creciente del sector en el comercio de productos intermedios (Sturgeon \& Memedovic, 2010) y el elevado número de etapas implicadas en la elaboración del producto (De Backer \& Miroudot, 2014).

\section{Metodología}

La metodología se basa en un enfoque integral. Se analiza en forma sistémica, los componentes de la cadena de valor para la fabricación de paneles fotovoltaicos en la empresa objeto de estudio, con la finalidad de difundir los contenidos entre los actores que intervienen en la cadena, en talleres participativos convocados a dichos efectos. La importancia de los actores radica en estructurar una gobernanza de dicha cadena, sobre la base de la gestión del conocimiento que permita respuestas rápidas y ágiles, a los cambios del entorno y problemas que se presenten en la búsqueda de un mejor desempeño.

Para el desarrollo de la presente investigación, se tuvo en cuenta como método general de la investigación científica el método dialéctico-materialista, que se despliega a lo largo de la investigación a través de métodos teóricos como:

- El análisis histórico (tendencial) y lógico, para analizar la evolución, desarrollo y esencia de la cadena de valor y la profundización en cuanto a los diferentes criterios de distintos autores relacionados con las diversas formas, métodos y vías para su realización, partiendo de Porter, la Comisión Económica para América Latina y el Caribe (CEPAL). Para terminar, se analizan las experiencias que existen en Cuba de esta temática, las cuales están centradas en industrias de tecnología avanzada (biotecnología e industria de medicamentos), el sector de la agricultura (el mango, el frijol y la producción tabacalera), forestal (servicios eco- 
sistémicos forestales), la industria alimentaria (cultivo del camarón y conservas de tomate) y el sector del turismo en Viñales para potenciar el desarrollo local, la cual tuvo un proceso de revisión de ocho meses durante el año 2019.

- Análisis y síntesis, es utilizado en la valoración crítica del marco teórico y contextual, relacionado con la cadena de valor y la profundización en cuanto a los diferentes criterios para su realización, se analizaron documentos para evaluar y clasificar el material bibliográfico recopilado que se relaciona con la cadena de valor, con el objetivo de analizar estas concepciones en Cuba y el diagnóstico del objeto de la investigación, como proceso de síntesis.

- Sistémico o estructural, para fundamentar la propuesta del procedimiento en el diseño de la cadena de valor en el proceso de toma de decisiones por la empresa, de forma tal que les permita un mejor desempeño de sus funciones y eficiencia de sus resultados. Para lograr lo anteriormente expuesto, se realizaron entrevistas a directivos como: la directora del departamento de contabilidad, el director del departamento de economía, el jefe de producción de la Unidad Empresarial de Base de paneles fotovoltaicos y el director general de la empresa. Todos fueron entrevistados individualmente, y cuentan con más de 15 años de experiencia como promedio, siendo por esta razón las personas más apropiadas para responder las preguntas relacionadas con la investigación abordada.

- Dicho proceso demoró alrededor de un año, pues a inicios de enero de 2020 se realizó todo de manera presencial y a finales del propio año de manera online por la existencia de la COVID-19 en Cuba; muchos de los datos e información fueron enviados por correo electrónico y vía telefónica.

- La investigación se encuentra en fase de validación y los resultados se mostraron parcialmente a la Dirección de Contabilidad y Economía para su revisión y ajustes de acuerdo a las exigencias de la entidad, y una vez mejorada la situación epidemiológica, mostrar a todos los directivos de la empresa y obreros de la producción.

En este sentido, la investigación forma parte de un estudio sobre las cadenas de valor en el país y como tal está en fase de desarrollo. Por lo tanto, los enfoques de clúster, cadena global y la innovación están en los planes futuros de los autores. Es por ello que la metodología utilizada se centró en las bondades de la cadena de valor como herramienta estratégica para la gestión empresarial. 


\section{Resultados}

\subsection{Propuesta de procedimiento para diseño de la cadena de valor}

Una vez analizados los trabajos referenciados anteriormente, en la figura 4, se expone la propuesta del procedimiento para el diseño de la cadena de valor en la fabricación de paneles fotovoltaicos, en la empresa de Componentes Electrónicos.

La propuesta consta de tres etapas: 1) estudio preliminar, 2) identificación de los principales procesos productivos en la fabricación de paneles fotovoltaicos y 3) desarrollo de la secuencia de pasos definidos para elaborar la cadena de valor. Se definen, para cada etapa, los métodos o herramientas para ejecutarlas, facilitando la búsqueda de ventajas competitivas, el diseño de la contabilidad de la cadena y la búsqueda de actividades generadoras de costo y valor, así como el cálculo de los indicadores financieros por cada uno de sus eslabones.

Figura 4. Propuesta del procedimiento para el diseño de la cadena de valor en la fabricación de paneles fotovoltaicos, en la empresa de Componentes Electrónicos

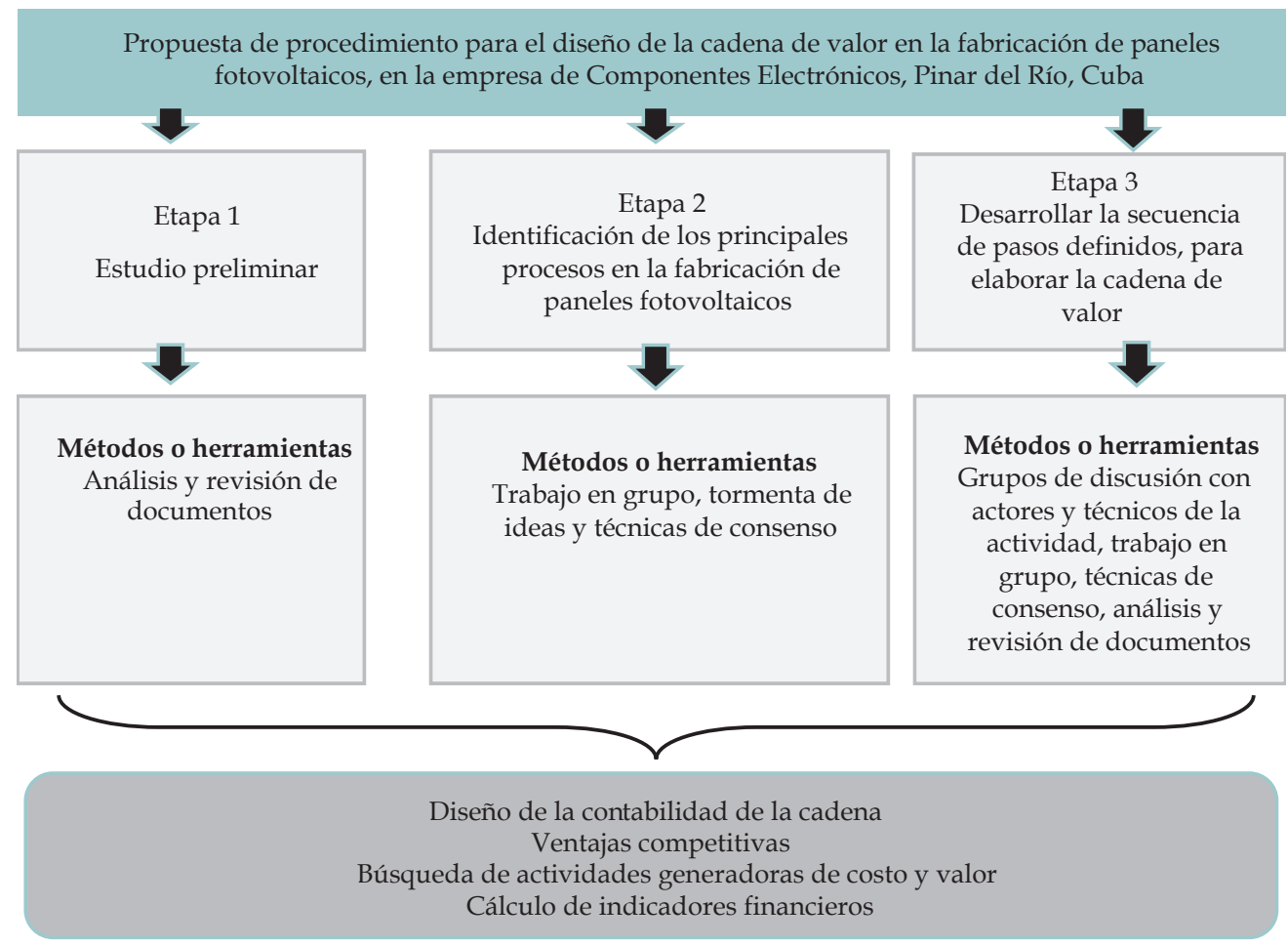

Fuente: elaboración propia. 


\section{Etapa I. Estudio preliminar}

La etapa es fundamental para lograr los objetivos propuestos y determinar los criterios e indicadores para diseñar el plan de trabajo. Se caracteriza por el conocimiento general del funcionamiento de la entidad.

Objetivo: seleccionar la información necesaria durante la investigación.

\subsubsection{Caracterización de la empresa bajo estudio}

Se muestra entonces el estudio preliminar y revisión de documentos, planteados en el procedimiento propuesto y en la metodología.

La empresa pertenece al Grupo de la Electrónica (GELECT) y está subordinada al Ministerio de Industrias (MINDUS). Participa en el proyecto The OPEC fund for inernational development (OFID), el cual está dirigido al crecimiento de la utilización de la energía solar en la generación de electricidad y calor, en correspondencia con la política nacional aprobada para el incremento del uso de las fuentes renovables de energía (FRE) y la eficiencia energética hasta el 2030, con alto impacto en el desarrollo social del país. El objetivo del proyecto está en correspondencia con el programa de inversiones destinado al cambio de la matriz energética y con el objetivo de alcanzar el $24 \%$ de participación de las FRE en el año 2030. Este programa incluye la instalación de $700 \mathrm{MW}$ en parques solares fotovoltaicos (PSF), conectados a la red eléctrica y ubicados en todas las provincias del país (González 2017, citado por Rojas et al., 2020a; Rojas et al., 2020b; Rojas et al., 2021). Se realizaron análisis de documentos como es el caso de los estados financieros, notas a los estados financieros de la entidad al cierre del año 2018 y 2019, su proyección estratégica hasta el año 2030, ficha de precios de los paneles fotovoltaicos, la implementación de las normas jurídicas en el Sistema Empresarial y la carta tecnológica del módulo fotovoltaico (CT0209).

González (2017) plantea que la empresa se beneficia con el financiamiento otorgado a través del Ministerio de Economía y Planificación generado por el proyecto (OFID), de aproximadamente 19.978.50 MUSD, a largo plazo, para la adquisición de las materias primas y componentes para fabricar 60.000 paneles fotovoltaicos anuales de 270 Watt $(\mathrm{W})$, con una relación de costo de 0,62 USD/Watt, de ellos: celda FV (62\%), vidrio (8 \%), marco (9\%), tedlar (6 \%), EVA (5\%) y un $10 \%$ para otros materiales: cinta de conex, silicona, caja de interconexión e insumos de fabricación. 
El país se ha propuesto alcanzar la capacidad de fabricación de módulos DSM$270 \mathrm{~W}$, los cuales definen su calidad por las características físicas (dimensiones [mm], el peso [kg], el número de celdas en serie y en paralelo) y las características eléctricas (potencia nominal o máxima, corriente de cortocircuito, entre otros), en un proceso que están siendo certificado y evaluado por la NC IEC-62 215:2012 en el Instituto de Energía Renovable de México.

\subsubsection{Caracterización general de proveedores y reguladores de la empresa de Componentes Electrónicos}

- EDIFRE de la Unión Nacional Eléctrica (UNE). Compra y comercializa los paneles fotovoltaicos en el mercado interno en divisa. Está dirigido a las inversiones de parques fotovoltaicos de la UNE destinados a inyectar a la red, con el objetivo de responder a la estrategia del país encaminada a diversificar la matriz energética nacional con las energías renovables. Participa de forma directa.

- COPEXTEL Ecosol. Compra, comercializa e instala los paneles fotovoltaicos en el mercado interno en pesos cubanos (CUP). Son sistemas aislados de inyección a la red. Fueron instalados hasta el momento en viviendas y comunidades rurales sin posibilidad de acceso a las redes eléctricas.

- Energomat. Compra los paneles fotovoltaicos dirigidos a potenciar la matriz energética nacional y uso de la energía limpia. Cada panel tiene un precio de venta de 294.98 moneda total por cada módulo. Cabe destacar que se han hecho exportaciones en frontera como es el caso de la compañía Resa Caribe de capital 100 \% español, a la cual se vendieron 200 paneles, y la empresa Construcción y Montaje, a la que se vendió un total de 1382 paneles, ambas ubicadas en Zona Especial de Desarrollo Mariel (ZEDM). Se está estudiando por parte de la empresa la exportación de paneles a Europa para la construcción de PSF y a Canadá para la instalación del módulo en viviendas.

- Proveedores internacionales de insumos y materias primas:

- CHC, HAIER, Beya Time, BNS, AVIC, Grumberck todos procedentes de China: estas compañías participan de forma indirecta en la venta de materias primas, equipos tecnológicos, piezas de repuesto, aditivos y ensamblaje de los paneles.

- Cuba Electrónica, proveedor nacional de insumos y materias primas: empresa importadora y exportadora perteneciente al OSDE GELECT 
del Ministerio de Industrias, participa de forma directa mediante la importación de materias primas, de equipos tecnológicos, piezas de repuesto, aditivos y ensamblaje de los paneles.

- Proveedores de servicios nacionales:

- AUSA: empresa encargada de abastecer los suministros generales como tuberías de polietileno, material de construcción en el mercado nacional, pallets de madera, nylon estirable, perfiles de aluminio, materiales para mantenimiento y la transportación de los paneles. La deficiencia en las relaciones con este proveedor radica en que no siempre se cuenta con la disponibilidad necesaria de camiones, y los arribos se producen, en determinadas ocasiones, los fines de semana no laborables, lo que provoca cuellos de botellas.

- ADIPEL: proveedor que participa de manera indirecta en la venta de insumos generales como envases, embalajes, etiquetas y manuales de funcionamiento. La deficiencia en las relaciones con este proveedor radica en que no siempre se cuenta con todos los insumos ni en la cantidad requerida.

- Transcontenedores: brinda servicios de manera indirecta para el traslado de contenedores con la materia prima del puerto hacia la fábrica.

Los proveedores entrevistados plantean que los procedimientos para la contratación e importación de los insumos y materias primas corren con cargas burocráticas y, debido a la existencia del bloqueo económico y problemas presupuestarios a nivel país, se ven obligados a recurrir al mercado chino, lo cual extiende los períodos de entrega de 180 hasta 260 días y por consiguiente afecta los resultados productivos.

- Reguladores:

- Ministerio de Economía y Planificación (MEP): tiene influencia en el marco político normativo a nivel nacional, en términos de economía.

- Ministerio de Finanzas y Precios (MFP): ejecuta y controla la política financiera-tributaria y de precios del Estado cubano.

- Ministerio de Ciencia, Tecnología y Medio Ambiente (CITMA): dirige, ejecuta y controla la actividad científica y tecnológica, la política ambiental.

- Ministerio de Industrias (MINDUS): controla y lleva a cabo las políticas industriales y estrategias para el desarrollo del país. 
Como bien se señala en esta etapa, son resultados preliminares, los cuales serán ampliados y perfeccionados una vez que se realicen los talleres de discusión con los actores, referidos en la tercera etapa.

\section{Etapa II. Identificación de los principales procesos en la fabricación de paneles fotovoltaicos}

Las medidas tomadas están en la propia reorganización de los flujos productivos, en modificaciones tecnológicas, remodelación de equipos, aumento de turnos de trabajo, el mejoramiento del aprovechamiento del tiempo-equipo y análisis del rendimiento productivo. Este último consiste en un control de la mano de obra mediante el procesamiento diario y por operario, de su quehacer productivo, evaluando su rendimiento y las pérdidas ocurridas, clasificándolas e incidiendo sobre ellas.

Objetivo: la recopilación de información de los procesos y subprocesos productivos.

Existe un primer resultado sobre los procesos productivos, tratado en trabajos anteriores, según Rojas et al. (2020a) y Rojas et al. (2021).

\section{Etapa III. Desarrollar la secuencia de pasos definidos, para elaborar la cadena de valor}

Se diseñará la cadena de valor de la empresa en la fabricación de paneles fotovoltaicos ya sea de forma directa, indirecta o inducida, a partir de sus relaciones con las demás actividades productivas y de servicios que se realizan.

Objetivo: concebir y comprender la cadena de valor de la empresa en la fabricación de paneles fotovoltaicos.

A partir del estudio de los procesos y actividades realizados mediante la revisión de documentos y observación, según López, (2016); Rojas (2017) y Domínguez et al. (2017), se estableció una secuencia de pasos para el diseño de la cadena de valor de la empresa.

- Paso 1. Formación del equipo de trabajo

Para diseñar el equipo de trabajo se tuvo en cuenta la estructura organizativa de la empresa, teniendo en cuenta la experiencia de los que integran la actividad industrial electrónica, fundamentalmente en la producción de paneles fotovoltaicos. 
- Paso 2. Discusión del plan de trabajo En este paso se establece el cronograma de trabajo a seguir, los aseguramientos necesarios (los insumos materiales y financieros necesarios), los responsables de las actividades a desarrollar y quiénes participan en cada una, para su acotación en el tiempo. Se planteó por los investigadores que el equipo multidisciplinario creado trabajaría en dos resultados esenciales que debían complementarse: el mapeo de las actividades productivas relacionadas con la fabricación de paneles fotovoltaicos y la representación general de la cadena de valor.

- Paso 3. Capacitación sobre la herramienta de la cadena de valor Tiene como objetivo fundamental la formación de capacidades básicas en los involucrados en el equipo de trabajo, para homogeneizar la comprensión del fenómeno a estudiar y la terminología para la que se va a trabajar desde los fundamentos teóricos y metodológicos que se encuentran en las bibliografías.

El trabajo se organizó en dos sesiones (presentaciones teóricas y talleres de discusión): la primera estuvo dedicada a los aspectos teóricos de la cadena de valor y la segunda a la gestión de procesos e indicadores financieros.

- Paso 4. Mapeo de las actividades productivas relacionadas con la cadena productiva de la fabricación de paneles fotovoltaicos, según Domínguez et al. (2017)

Se tomó como insumo todo el proceso llevado a cabo para la identificación de los procesos estratégicos, claves y de apoyo, generales para toda la empresa; técnicas empleadas para identificar los procesos fundamentales que se desarrollan al interior de la unidad productiva destinada a la fabricación de paneles fotovoltaicos; así como la descripción del objeto social empresarial, la misión y clasificación de las actividades. Todo ello basado en los fundamentos teóricos que se abordaron, y teniendo como premisa la capacitación concretada en el paso 3.

- Paso 5. Definición de las entradas de la cadena

Se definen las entradas de la cadena, conocidas como inputs a los procesos, subprocesos y actividades, identificados y mapeados con anterioridad. Se determinan los elementos que entran al eslabón denominado "logística de entrada" para las actividades primarias, y el eslabón llamado "aprovisionamiento" dentro de las actividades de apoyo, se hace referencia a todas las entradas posibles de recursos (materiales, humanos, financieros, información y tecnológicos) que intervienen en el proceso de transformación y creación de valor. 
Definición de las entradas:

- Materias Primas y materiales fundamentales: EVA, tedlar, silicona, celdas, ribbon, flux, vidrio templado pequeño, erlenmeyer, tapón con dos orificios, soporte universal, papel de filtro, papel de celulosa, baño termostatado, cinta de cobre, etiqueta con número de serie, alcohol (etanol), papel para limpieza, hilo de estaño, cinta adhesiva, mantas de teflón, guantes reforzados, membrana de silicona, complete aluframe set, aluframe long side, aluframe short side, cinta de enmarcado, detergente líquido, brocha, resina 1521 Tipo, boquilla (nebulizador modelo MC 10-24), esquinero para marco de aluminio, fleje plástico, paleta, tóner para etiquetadora, grapas lisas y nylon estirable.

- Medios: alumbrado local, computadora, climatización unidades exteriores, climatización unidades interiores, cautín, alumbrado verificación sándwich, laminador 5, laminador 6, laminador 4 (chino), compresor, pistola para aplicar jet melt, dispensador de resina.

- Recursos tecnológicos: simulador solar (Solar Cell Tester BSCT102), balanza analítica, balanza técnica, máquina de soldadura automática, desumificador, máquina de formación de tiras, máquina de corte de EVA y tedlar, equipo de prueba de aislamiento, simulador solar y equipo de electroluminiscencia con computadora acoplada.

- Recursos financieros: créditos bancarios, estudios de factibilidad, desembolsos, cheques y órdenes de pago.

- Recursos de información: asesoría técnica y publicidad del producto.

- Paso 6. Definición de los eslabones principales

Se establecen los eslabones que conforman el componente de "operaciones" dentro de la cadena de valor, determinándose anteriormente las entradas. Los eslabones fundamentales son cinco: control de entrada, formación de tiras en la máquina de soldadura automática, formación del sándwich e inspección del sándwich con equipo de electroluminiscencia y el laminado del sándwich. Cuando trimado de los bordes, colocación del marco (encintado y pre enmarcado, colocación automática del marco), pegado de la caja de conexión, prueba de aislamiento, inspección del módulo enmarcado con equipo de electroluminiscencia, verificación final del módulo, llenado de la caja de conexión, terminado del módulo, control final y embalaje son considerados procesos operativos, desde el punto de vista de cadena de valor genérica están comprendidos en la logística externa. 
- Paso 7. Definición de los eslabones de apoyo En este paso se definen los eslabones de apoyo, que en la cadena de valor se hacen coincidir con las actividades de apoyo, ya que se consideran: la infraestructura, tanto física como técnica y tecnológica, para el desarrollo de los procesos, incluyendo la dirección general, contabilidad, finanzas y planificación, la calidad y los recursos con que cuentan las formas productivas; el capital humano y la comercialización propiamente de apoyo a partir de entradas al proceso definidas en el paso 5.

- Paso 8. Definición de las salidas de la cadena

Se consideran tanto los elementos que conforman la logística de salida (verificación final del módulo, llenado de la caja de conexión, terminado del módulo, control final y embalaje), así como el proceso de comercialización de producciones en la fabricación de paneles fotovoltaicos. Como salidas globales más importantes está el margen resultante de la diferencia entre valor generado y costo incurrido a lo largo de la cadena (expresados en términos de utilidades netas y valor agregado bruto y, por otra, los clientes satisfechos). Aquí se logra cerrar el ciclo productivo de la empresa, hasta que entrega los paneles fotovoltaicos a EDIFRE de la Unión Nacional Eléctrica (UNE).

- Paso 9. Identificación de los beneficiarios de la cadena

Tiene como objetivo identificar los beneficiarios directos de la cadena, los que obtienen la producción de la actividad principal que se produce.

- Como beneficiario principal se tiene: EDIFRE de la Unión Nacional Eléctrica (UNE).

- Otros beneficiarios con personalidad jurídica: Copextel Ecosol, Energomat.

- Personas naturales: las viviendas y comunidades rurales.

- Paso 10. Representación gráfica de la cadena.

Como objetivo se tiene que graficar la cadena de valor de la empresa, integrando los análisis realizados a lo largo de todo el proceso analizado, según se muestra en la figura 5. 


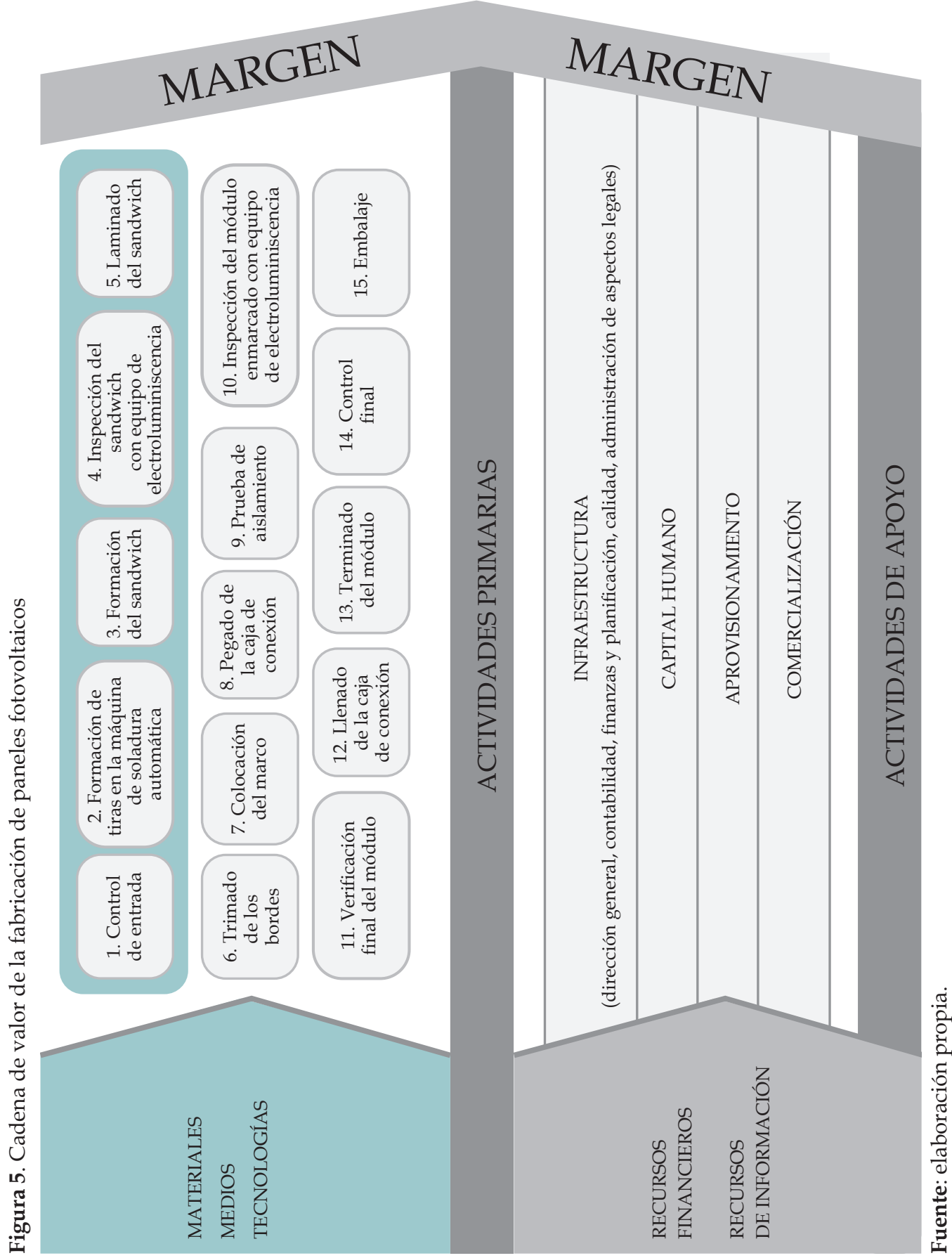




\section{Discusión}

En la Agenda 2030 y los Objetivos de Desarrollo Sostenible (ODS) asociados a ella, aprobados ambos por la Organización de Naciones Unidas (ONU), se tiene como principio transformar el paradigma de desarrollo actual en uno que lleve por la vía del desarrollo sostenible, inclusivo y con una visión de largo plazo. Cuba se posiciona en la urgencia de la transformación de su matriz energética, para disminuir el consumo de combustibles importados para la generación y aumentar la eficiencia en su utilización. La actualización del modelo económico cubano incluye, en el cumplimiento del programa aprobado hasta 2030, el desarrollo de las fuentes renovables y el uso eficiente de la energía.

García (2020) enfatiza que la Isla tiene un plan gubernamental de instalar 700 megawatt (MW) para 2030, por lo que con la actual producción anual la empresa apenas cubriría cerca de un tercio de las necesidades nacionales, pues a partir de los resultados del Censo de Población y Viviendas de 2012, en la actualidad existen 20.000 viviendas sin acceso a la electricidad, lo que generó la conformación de un proyecto gubernamental para facilitar ese servicio a través de paneles solares cuya vida útil está estimada en 20 años. Las fuentes renovables de energía han generado hasta el 2019, 687.000 megawatt-hora (MW/h), lo que representa un ahorro de 178.000 toneladas de combustible y cuando se instalen todas las tecnologías pertinentes concebidas para el año 2030, se prevé obtener una generación energética en el país de 7000 gigawatthora $(\mathrm{GW} / \mathrm{h})$, lo que permitiría ahorrar 1.800 .000 toneladas de combustible, aproximadamente.

Rojas (2021) plantea que la empresa se encuentra en pleno proceso de reanimación y redimensionamiento; en la cadena de valor, aún no definida por la empresa, se identificaron los principales cuellos de botella en el proceso productivo, así como en los flujos tecnológicos, que se evidencian en una serie de deficiencias en su funcionamiento. Dicho autor identifica un conjunto de problemas en la empresa Componentes Electrónicos, los cuales podrían ser solucionados con el diseño de la cadena de valor propuesta, a saber:

1. No se analizan periódicamente las desviaciones de las materias primas.

2. No tienen definidas las actividades generadoras de costo y valor en sus procesos en función de la cadena.

3. No están determinados los eslabones de la cadena, solo la lógica de los procesos productivos. 
4. La empresa tiene definidos los proveedores, clientes y reguladores, pero no identificados como actores de la cadena.

5. Problemas tecnológicos debido a las insuficiencias de mantenimientos como:

- Deficiencia en el sistema de calentamiento y desmonte de las cadenas de los laminadores.

- Deficiencias en el soporte de royo y guías de ribbon de la máquina soldadura automática.

- Deficiencias en el rodamiento de la correa del simulador solar.

6. Dificultades en la ampliación de la capacidad productiva y entorpecimiento del flujo productivo con capacidades de almacenamiento limitadas.

7. No se identifican las posibles fuentes de ventajas competitivas, que es fundamental para diseñar una oferta acertada para su mercado, meta o público objetivo.

8. No existe know-how para añadir valor a la producción con destino a la exportación.

9. Dificultades en el proceso de contratación de los bienes intermedios y demoras de la importadora.

10. No cuenta con una buena proyección económico-financiera para alcanzar en el futuro valores que eleven su eficiencia.

11. Los clientes no cuentan con una infraestructura logística de transporte adecuada para asumir los incrementos productivos.

12. Escenario tecnológico muy dinámico que requiere de constantes inversiones para mantener a la industria actualizada.

13. Inestabilidad para obtener financiamientos internos y externos a largo plazo y amplia dependencia de la economía cubana del exterior.

En las dos primeras etapas del procedimiento, existen resultados preliminares, referidos en la descripción de las mismas, que son la base para el desarrollo de la tercera etapa, todavía sin ejecutar. Esta tercera etapa está relacionada fundamentalmente con la implementación de la cadena propuesta, lo que conlleva la creación de grupos de trabajo, talleres de capacitación, la retroalimentación del diseño propuesto y, lo más importante, que los funcionarios, directivos y trabajadores comiencen a trabajar con este enfoque.

Aunque los principales directivos no están ajenos a esta investigación, hay que esperar que se puedan flexibilizar las medidas para el control de la pandemia de la COVID-19 en Cuba, para seguir con la tercera etapa de este procedimiento. 


\section{Conclusiones}

La producción de paneles fotovoltaicos tiene una gran importancia para la economía del país, por la necesidad de su contribución a la energía limpia, su uso eficiente y al cambio del estado actual de la dependencia energética de las importaciones de combustible fósil. En este sentido, uno de los objetivos de la agenda 2030 nacional, es lograr que el $24 \%$ del consumo energético en el país sea de fuentes renovables de energía. Sin el fortalecimiento del encadenamiento productivo, el proceso de creación de valor y las fuentes de competitividad, en la generación de energía fotovoltaica, será muy difícil lograr ese objetivo nacional.

El diagnóstico realizado en la empresa objeto de estudio permitió caracterizar el modelo de gestión actual, determinándose la estructura de las actividades que conforman la cadena de valor, la identificación de los procesos y subprocesos, evidenciándose que no utiliza la cadena de valor como instrumento para analizar y diagnosticar.

El procedimiento propuesto tributa al diseño de los procesos contables y el cálculo del costo por cada uno de los eslabones de la cadena de valor, ayudando a la formalización de los acuerdos financieros, comerciales y de cooperación, así como al diseño de estrategias que permitirán eficiencia en las operaciones gerenciales y financieras. El mismo se encuentra en fase de validación e implementación en la referida empresa, una vez que se hayan desarrollado los pasos de la tercera etapa, lo que permitirá la creación de una red de valor dentro de su sistema de información, que mejore la competitividad, lo cual demuestra la importancia tanto en el plano teórico como empírico del diseño de la cadena de valor en las organizaciones.

No existen antecedentes en el país de propuestas de cadena de valor en ningún estadio, ya sea diseño, validación o implementación, en el sector industrial, por lo que el trabajo constituye un aporte en ese sentido. Continuará su desarrollo por el interés en primer lugar de la empresa objeto de estudio de utilizar esta herramienta como parte de su gestión administrativa y estratégica.

Para estudios futuros la realización del diagnóstico cualitativo de la situación a investigar y el método de medición para cuantificar los impactos de la realización del diagnóstico al procedimiento para el diseño de la cadena de valor, se tomará como base el método de Preferencia, como evaluación el criterio de expertos para validar el problema general de la investigación, empleando el Software Social Sciences Program Stadistic (SSPS). 


\section{Referencias}

Anaya, B. (2015). Las cadenas productivas con impacto económico y social: el caso de los cítricos en Cuba. Economía y Desarrollo, 154(1), 105-117.

Antúnez Saiz, V. I., \& Ferrer Castañedo, M. (2021). Metodología para el análisis de cadenas productivas en Cuba: el caso de Agrocadenas como proyecto innovador. Estudios del Desarrollo Social: Cuba y América Latina, 9(2), 33-49.

Baldwin, R., \& Venables, A. (2013). Spiders and snakes: Offshoring and agglomeration in the global economy. Journal of International Economics, 90(2), 245-254.

Blanco, L. (2013). Hirschman: un gran científico social. Economía Institucional, 15(28), 47-64.

Bonmatí Martínez, J. (2011). El valor de una empresa y la creación de valor en esa empresa. Madrid: AECE.

De Backer, K., \& Miroudot, S. (2014). Mapping global value chains. Global value chains and world trade: Prospects and challenges for Latin America. Santiago: ECLAC, 2014. LC/G. 2617-P, 43-78.

Domínguez Junco, O., Rojas Hernández, D., Gómez Hernández, J., \& Medina Peña, R. (2017). Metodología para gestión contable de los servicios ecosistémicos forestales con enfoque de cadena de valor. Revista científica Agroecosistemas, 5(1), 71-78.

Espinal, F. (2004). Cadenas productivas, experiencias y estrategias para el desarrollo de la competitividad en Colombia. Ponencia presentada en el Seminario sobre Alianzas, Cadenas Productivas y Competitividad para un Desarrollo Sustentable. Monterrey.

Espinosa, E. G., \& Díaz, E. (2021). Consideraciones sobre la cadena de valor del camarón en Cuba. Apuntes para un diagnóstico. Cofin Habana, 15(1).

García, A. (20 de agosto de 2020). La energía que renueva a Cuba. Granma. Recuperado de https:/ / www.granma.cu/cuestion-de-leyes/2019-11-28/ la-energia-que-renueva-a-cuba-28-11-2019-00-11-44

García, J. (2007). Las cadenas productivas y el cluster turístico, factores dinamizadores del desarrollo local. Una aproximación a la realidad del municipio Yaguajay. Economía y Desarrollo, 142(2), 172-194.

González, I. (20 de junio de 2017). Proyecto OFID: Energía Solar para la Generación de Energía y Calentamiento de Agua, Consejo de dirección de la empresa de Componentes Electrónicos, Grupo de la electrónica, Ministerio de Industrias, Pinar del Río, Cuba. 
Gorbaneff, Y., \& Restrepo, A. (2007). Determinantes de la integración vertical en la cadena de distribución de combustible en Colombia. Cuaderno de Administración, 20(33), 125-146.

Gottret, M. (2007). Gestión de Cadenas Productivas. La Paz: Impresiones Sagitario. International Accounting Standards Board. (2006). Norma Internacional de Información Financiera No. 7, Instrumentos financieros: Información a revelar.

International Accounting Standards Board. (2006). Norma Internacional de Contabilidad No. 1, Presentación de Estados Financieros.

Isaza, J. G. (2005). Cadenas productivas: enfoques y precisiones conceptuales. Sotavento, 11, 8-25.

Jiménez Barbosa, W. G., \& Montenegro Martínez, G. (2014). Las relaciones entre los actores de una red de salud. Cienc Tecnol Salud Vis Ocul, (2), 107115. https://doi.org/10.19052/sv.3299

López, A. (2011). Cadenas globales de producción, redes globales de producción y competitividad regional. Mundo Económico y Empresarial, 11.

López, A. C. (2016). Propuesta de cadena de valor de la producción tabacalera en la Empresa de Acopio y Beneficio de Tabaco "Hermanos Saíz" de San Juan y Martínez [Tesis de pregrado, Universidad de Pinar del Río, Pinar del Río, Cuba]. Repositorio Institucional.

Lladós Masllorens, J., Meseguer Artola, A., \& Vilaseca Requena, J. (2018). La cadena global de valor en la industria electrónica. Inv. Econ, 77(304), 135-170. Mandal, S. (2012). Supply chain performance: review of empirical literature. Romanian Review of Social Sciences, 3, 24-34.

Manzo Martínez, M. A. (s. f.). El análisis de la cadena de valor como fuente de ventajas competitivas en las empresas exportadoras de zarzamora en México. Red Internacional de Investigadores en Competitividad Memoria del IX Congreso.

Máttar, J., y Padilla, R. (s. f). Fortalecimiento de cadenas de valor agroalimentarias en Cuba. Los casos de las conservas del tomate y el camarón de cultivo. Comisión Económica para América Latina y el Caribe (CEPAL), Naciones Unidas.

Menoya, S. (2015). Modelo de gestión del turismo desde el gobierno local para municipios con vocación turística basado en el enfoque de cadena de valor (Tesis doctoral). Universidad de Pinar del Río, Pinar del Río, Cuba. Repositorio Institucional. 
Ministerio de Finanzas y Precios. (2018). Norma Específica No. 12, sobre la Contabilidad de Gestión. Gaceta Oficial de la República de Cuba 2019-231O15.

Naciones Unidas. (2016). Objetivos de Desarrollo Sostenible de la ONU.

Nogueira de Morais, I. (2012). Global productive chains and value added: China's position in the electronic industry. The Perspective of the World Review, 4(3), 5-44.

Nova, A., Prego, J. C., \& Robaina, L. (2020). El encadenamiento productivovalor en Cuba. Antecedentes y actualidad. Proyecto APOCOOP. Estudios del Desarrollo Social, 8(1).

OECD (2012) [Organisation for Economic Co-operation and Development]. Mapping global value chains [Report TAD/TC/WP/RD(2012)9]. Trade and Agriculture Directorate, Trade Committee, oecd, París, Francia.

ONUDI. (2011). Industrial Value Chain Diagnostics: An Integrated Tool. Viena. Padilla Pérez, R., \& Oddone, N. (2016). Manual para el fortalecimiento de cadenas de valor, Ciudad de México. Comisión Económica para América Latina y el Caribe (CEPAL), Naciones Unidas.

Partido Comunista de Cuba (PCC) (2012). Lineamientos de la Política Económica y Social del Partido y la Revolución. VI Congreso, Editora Política: La Habana Partido Comunista de Cuba (PCC) (2016). Lineamientos de la Política Económica y Social del Partido y la Revolución, VIl Congreso. Editora Política: La Habana Plasencia, M. (2006). Influencia de los corredores económicos en la producción de limón de exportación en la región de Piura. Journal of Economics, Finance and Administrative Science, 11(20), 153-175.

Porter, M. (1985). Competitive Advantage: Creating and Sustaining Superior Performance. New York. Free Press.

Porter, M. (1993). Ventajas competitivas: creación y sostenimiento de un desempeño superior. México: Continental.

Rojas Hernández, D. (2021). Metodología para diseñar la cadena de valor en la fabricación de paneles fotovoltaicos como soporte en la gestión financiera en la empresa de Componentes Electrónicos, Pinar del Río (Tesis de maestría). Universidad de La Habana, La Habana, Cuba.

Rojas Hernández, D., Espinosa Martínez, E. G., Rojas Hernández, D., \& Pelegrín Mesa, A. (2020a). Perfeccionamiento al proceso de acumulación y cálculo del costo en la fabricación de paneles fotovoltaicos. Ide@s CONCYTEG, 15(278), 33-48.

Rojas Hernández, D. (2017). Metodología para la gestión contable de los Servicios Ecosistémicos Forestales (SEF), con enfoque de cadena de valor en 
la Empresa Agroforestal de Pinar del Río (Tesis de pregrado). Universidad de Pinar del Río, Pinar del Río, Cuba.

Rojas Hernández, D., Espinosa, E. G., Pelegrín, A., \& Menoya, S. (2021). Los procesos en la fabricación de paneles fotovoltaicos, una revisión desde la perspectiva de generación de valor empresarial. Artículo científico (Manuscrito no publicado). San José: Costa Rica.

Rojas, D., Pelegrín, A., Cabrera, N., \& Rojas, D. (2020b). Procedimiento de trabajo administrativo para el cálculo del costo de paneles fotovoltaicos. AvaCient, IX(2), 89-100.

Sturgeon, T. J., \& Kawakami, M. (2011). Global value chains in the electronics industry: Characteristics, crisis, and upgrading opportunities for firms from developing countries. International Journal on Technological Learning, Innovation and Development, 4(1-3), 120-147.

Sturgeon, T. J., \& Memedovic, O. (2010). Mapping global value Chains: Intermediate goods trade and structural change in the world economy [Working Paper no. 05/2010]. Development Policy and Strategic Research Branch, United Nations Industrial Development Organization (unido), Viena, Austria.

Timmer, M. P., Erumban, A. A., Los, B., Steherer, R., \& de Vries, G. J. (2014). Slicing up global value Chains. Journal of Economic Perspectives, 28(2), 99-118. Van Der Heyden, D., \& Camacho, P. (2006). Guía metodológica para el análisis de cadenas productivas. Centro Internacional para el desarrollo, Lima Perú. Vinci, A. (2014). Hacia una Gestión con Enfoque de Cadena. Conceptos Básicos e Instrucciones para el Diagnóstico. La Habana.

Vivanco Aranda, M., Martínez Cordero, F. J., y Taddei Bringas, I. C. (2010). Análisis de competitividad de cuatro sistema-producto estatales de tilapia en México. Estudios sociales (Hermosillo, Son.), 18(35), 165-207.

Wisner, J. (2003). A structural equation model of supply chain management strategies and firm performance. Journal of Business Logistics, 24(1), 1-25. https:/ / doi.org/10.1002/j.2158-1592.2003.tb00030.x

(C) 2021 por los autores; licencia otorgada a la revista Escritos Contables y de Administración. Este artículo es de acceso abierto y distribuido bajo los términos y condiciones de una licencia Atribución-No Comercial 4.0 Internacional (CC BY-NC 4.0) de Creative Commons. Para ver una copia de esta licencia, visite https://creativecommons.org/licenses/by-nc/4.0/ 